\title{
Improved Processing of Carbon Nanotube Yarn
}

\author{
Chaminda Jayasinghe, ${ }^{1}$ Trent Amstutz, ${ }^{1}$ Mark J. Schulz, ${ }^{2}$ and Vesselin Shanov ${ }^{1}$ \\ ${ }^{1}$ Department of Chemical and Material Science Engineering, University of Cincinnati, Cincinnati, OH 45221-0012, USA \\ ${ }^{2}$ Department of Mechanical Engineering, University of Cincinnati, Cincinnati, OH 45221-0072, USA
}

Correspondence should be addressed to Vesselin Shanov; Vesselin.Shanov@uc.edu

Received 22 October 2012; Accepted 10 March 2013

Academic Editor: Qian-Ming Gong

Copyright (C) 2013 Chaminda Jayasinghe et al. This is an open access article distributed under the Creative Commons Attribution License, which permits unrestricted use, distribution, and reproduction in any medium, provided the original work is properly cited.

\begin{abstract}
We compared mechanical and electrical properties of carbon nanotube (CNT) yarns formed from four different spinning methods. In these methods, a yarn was spun from two aligned CNT arrays. CNT yarns fabricated from each method were tested quantitatively through the mechanical and electrical properties and reported. This improvement is considered to be caused by multiple factors, such as reduction of the yarn diameter, densification, water evaporation, and CNT orientation. The best electrical and mechanical property of CNT yarn was observed from the fourth spinning method where heating and tension during spinning were applied. The introduced yarn spinning methods are appropriate for continuous mass production of high strength carbon nanotube yarns with controlled diameter, strength, and electrical conductivity.
\end{abstract}

\section{Introduction}

Carbon nanotubes (CNTs) are considered one of the strongest and stiffest materials known. They have unique atomic structure with interesting related properties such as high tensile strength and Young's modulus, high aspect ratio, and good electrical and thermal conductivities. These unique properties open up various applications in different areas, for example, in advance electronics, biotechnology, and nanomechanics.

Research efforts have been carried out to fabricate macroscopic threads and yarn structures to make the use of the CNTs unique properties. However there are technical challenges to transfer individual CNT properties into spun yarn structures because CNT bundles tend to slide in yarn microstructures. Therefore may be promising techniques have been implemented to improve the strength of the CNT yarn. One practical application is polymer dip coating which will improve the mechanical properties of CNT yarns structures but it will not help to improve the electrical properties of the yarn. Other is high temperature annealing which will help to improve the electrical and thermal properties of the CNT yarn. However, the disadvantage of thermal annealing is high cost and brittle behavior of the yarn structures.
There are mainly two methods of CNT yarn production: liquid-state spinning and solid-sate spinning. Most synthetic fibers are created from a concentrated, viscous liquid. In liquid based spinning, CNTs are dispersed into fluids and either extruded or coagulation spun into fibers [1-3]. The major issue with applying this approach to CNTs is that there is a relatively high fraction of remaining polymer and using of short CNTs, which limits the overall strength and electrical and thermal conductivity of the produced fiber [4]. The solidsate spinning can be done in two methods. One is spinning CNT yarns directly from a furnace using floating catalyst [5]. The second method is substrate base spinning. Spinning directly from a furnace is a very efficient approach for continuous production of yarn that has good properties $[6,7]$. Limitations of this method are the nanotubes used relatively short and there are catalyst impurities deposited in the final product.

Cotton and wool are naturally made fibers that are made by solid-sate spinning. In solid state spinning, CNTs are assembled into either yarns or sheets without the use of any chemical additives. This process is practiced by a handful of research groups $[2,5,8-11]$. The posttreatment methods play an important role to improve the properties of CNTs. Zhang et al. showed substantial increase in the strength of 


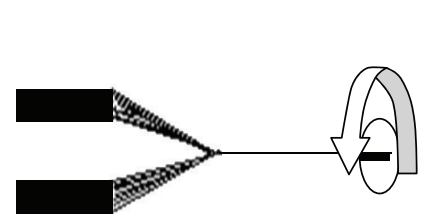

(a)

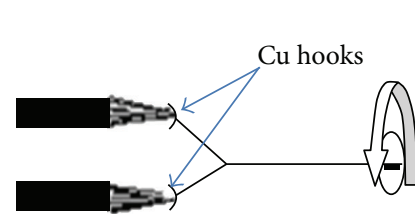

(b)

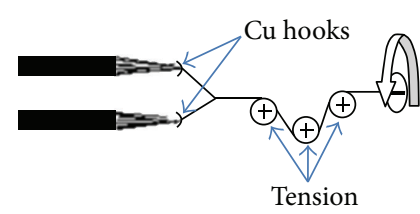

(c)

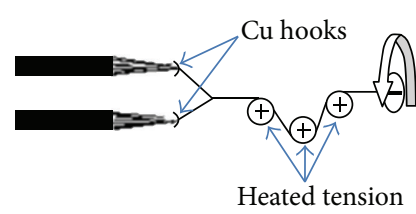

(d)

FIGURE 1: Schematic of the four different spinning methods (Methods 1 to 4) illustrated in the schematic corresponding to figures (a) to (d).

the CNT threads after infiltration with a polymer [10]. Zhu et al. reported that CNT yarn load capacity can be improved by postspun twisting which is affected by two factors: bringing the CNTs into closer contact with one another and therefore enhancing the van der Waals forces and higher radial compressive stress [5]. Other posttreatments involve densification with organic solvents such as ethanol and acetone, or heating the thread at high temperatures by applying an electric current [12]. Those posttreatment techniques require extra processing time [13-15]. In this paper, four improved methods for spinning CNT yarns are presented. The mechanical and electrical properties of CNT yarns made by each method are compared.

\section{Experimental Procedure}

We introduce four comparative methods of CNT yarn spinning. Spinnable carbon nanotube arrays were grown on silicon wafers using Chemical Vapor Deposition (CVD) at $750^{\circ} \mathrm{C}$ for 30 minutes [16]. CNT arrays about $325 \mu \mathrm{m}$ in height were spun into yarns at about a $2000 \mathrm{rpm}$ twisting rate and a $324 \mathrm{~mm} /$ minute pulling speed.

Based on Method 1 (spinning yarn from two CNT arrays), two CNT forests were drawn out forming two ribbons which were directly spun into a two-ply thread without any further treatment (Figure 1(a)). This spinning technique has been used by several other groups $[2,5,8-11]$ and served in our experiments as a reference (baseline) to which all the other techniques were compared in the present work. Method 2 (two arrays with cupper hooks) was designed to employ two copper hooks which applied small tension force to the ribbons prior to spinning (Figure 1(b)). This arrangement forced the ribbons to condense and spin into low tension threads before they were combined into the final thread. Method 3 (two arrays with cupper hooks and tension rods) improves the second method by using the same hook system but also implementing several tensioning rods along the spun yarn (Figure 1(c)). The rods applied tension to the thread in order to increase the interactions between the CNTs and to enhance the van der Waals forces between the individual carbon nanotubes. The intention of this arrangement was to increase the strength and decrease the resistivity of the thread. Method 4 (two arrays with cupper hooks and heated tension rods) is a modification of the third one, where the tensioning rods were modified to heat the thread simultaneously while applying tension (Figure 1(d)). This the method we call heating and tension spinning (HTS).

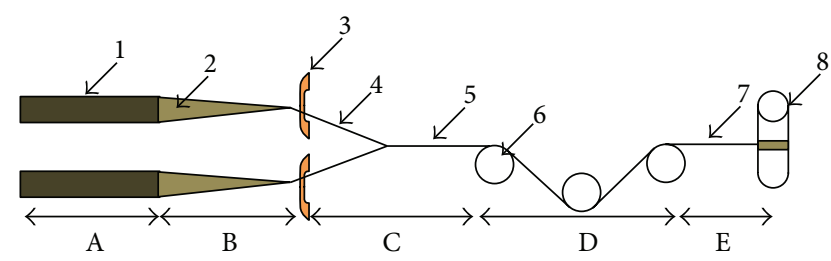

FIgURE 2: Schematic of the setup used in the proposed improved spinning process: A: CNT forest; B: web formation zone; C: partially twisted thread and yarn formation zone; D: tension and heat zone; E: twisting zone; (1) spinnable CNT forest; (2) CNT web; (3) copper hook; (4) partially twisted thread; (5) low twisted yarn; (6) heated rod for applying tension; (7) high twisted yarn; (8) spool.

The produced yarns were collected on spools. The samples were tested to determine their electrical resistivity and ultimate tensile strengths. Scanning electron microscopy (SEM) was used to measure the diameters of the tested yarns. The tensile strength measurement was conducted with a 3345-Instron stress-strain instrument. A four-probe setup was employed to determine the electrical properties of the yarn using a Keithley 6220 current source and a 2182A nanovoltmeter. All the tests were conducted multiple times to verify consistency of the acquired data.

\section{Results and Discussion}

3.1. Production of Carbon Nanotube Yarn. Currently, dry spinning of CNTs is conducted by twisting the formed web while pulling CNTs from a single substrate $[2,5,8-11]$. This process can be improved by introducing different spinning techniques. Recently, $\mathrm{Hu}$ et al. tested a modified spinning process attempting to improve the mechanical properties of CNT yarns [17]. They formed CNT threads under tension spinning in a high volume heated environment. Here we introduced resistive heating tension rods which can be used as tension rods as well as heat sources. Therefore the process is really simple and cost effective. This paper also provides a detailed characterization and explanation of the improved spinning process. Figure 2 shows the main components of the improved spinning setup. The main steps in the process for spinning thread are described next.

3.1.1. Web Formation. CNTS initially pulled from the forest form a web. As the CNT bundles are pulled away from the forest they form a long "line" in which all the CNT centerlines are 


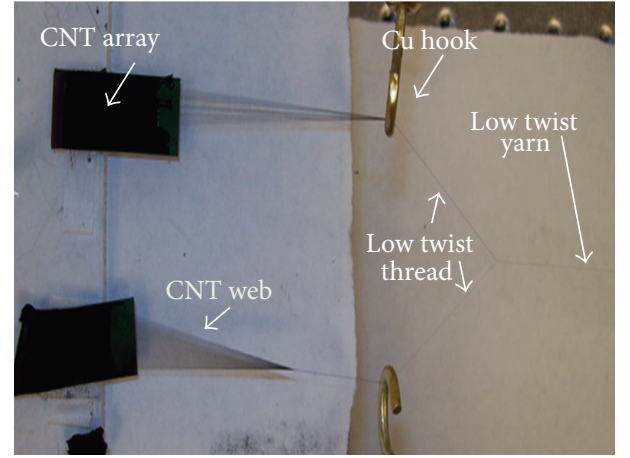

(a)

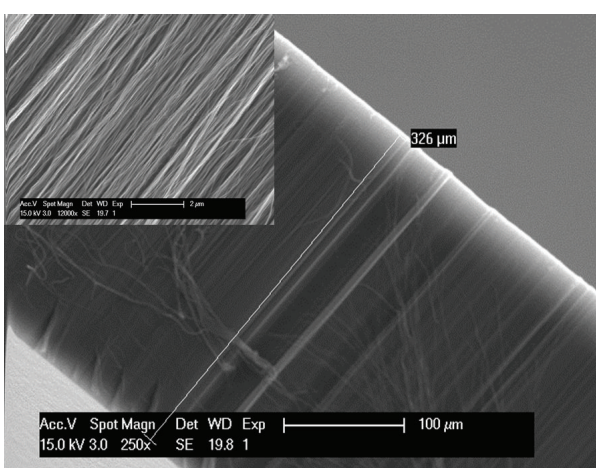

(c)

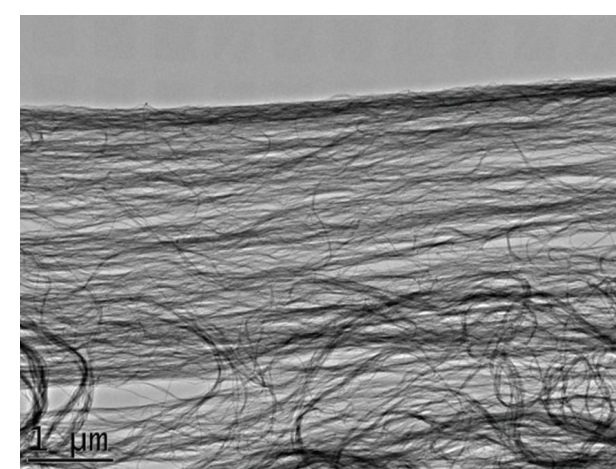

(b)

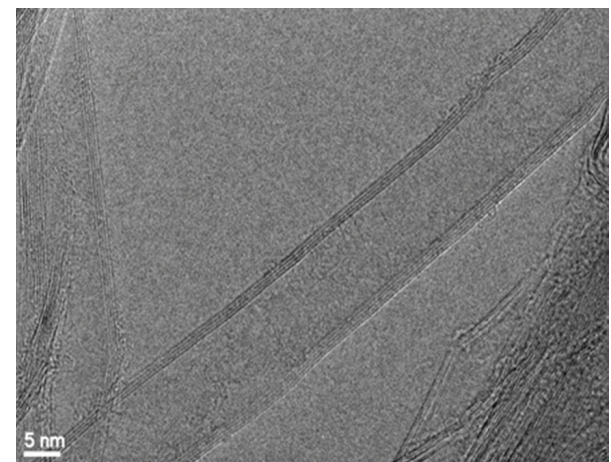

(d)

FIGURE 3: (a) Two CNT arrays spun into a yarn using the improved spinning method, (b) TEM image of CNT web, (c) SEM image of aligned CNT array (inset shows the forest cross-section at high magnification) and (d) HRTEM image of individual CNT with diameter $\sim 15 \mathrm{~nm}$.

aligned and parallel. The CNT web is orthogonal to the initial nanotube direction in the array and parallel to the substrate surface. The web must be kept stable during the spinning process in order to yield well-formed yarn. As shown in Figure 3(a), smooth copper hooks are placed between the first tension rod and the substrates. This improves the contact between the loosely attached CNT bundles within the web. The portion between the CNT forest and the copper hooks is the CNT web, and just after the copper hook it is transformed into a low twist thread. During the spinning process, those low twisted threads are combined to form one yarn. The joining point is called the convergence point. The convergence point is located before the first tension rod. The portion of the yarn shown in Figure 2 at position 5 is called low twisted yarn. In general, the web is "fluffy" and for this reason it needs to be compacted before spinning. Transmission electron microscopy (TEM) images show that the CNT web consists of poorly aligned and randomly orientated CNT bundles (Figure 3(b)). The thickness of the web ranges from $50 \mathrm{~nm}$ to $100 \mathrm{~nm}$. According to the capstan equation from the rope theory, when yarn passes through a pulley, the change in tension and the corresponding radial pressure on the yarn can be written as follows $[17,18]$ :

$$
\begin{gathered}
\frac{T_{2}}{T_{1}}=e^{\mu \varphi}, \\
N_{1}=\frac{T_{2}}{\mu}\left(e^{\mu \varphi}-1\right),
\end{gathered}
$$

where $T_{1}, T_{2}, \mu, \varphi$, and $N_{1}$ are the low incoming tension and high outgoing tension, the frictional coefficient, the contact angle, and the radial pressure on the yarn, respectively.

3.1.2. Thread Formed under Tension. Figure 4 illustrates the arrangement of two copper hooks which apply a small initial tension force to the ribbons prior to the spinning. This force helps the ribbons to condense and spin together slightly before they combine into partially twisted yarn. This part of the fiber is called "low twisted yarn." When the CNT web passes through the copper hooks, tension increases according to the capstan equation (1). This tension causes convergence of the web which is observed at a point close to the copper hooks. Further, Figure 4 displays the system using spinning under tension, where $T_{w}, T_{1}, T_{5}, \Omega_{1}$, and $\Omega_{5}$ are the web tension (incoming and outgoing after copper hook), final tension (near the CNT yarn winding spool), twisting of preformed thread, and twisting of final yarn, respectively. As the yarn passes through different stages, it is subjected to gradually increasing tension. The magnitude of the tension for a 3-tension rod arrangement increases accordingly: $T_{w}<$ $T_{1}<T_{2}<T_{3}<T_{4}<T_{5}<T_{3}<T_{4}<T_{5}$. Thus, for the tested spinning system, the change in tension after the last tension rod $\left(T_{5}\right)$ and the corresponding final radial pressure $\left(N_{5}\right)$ on the yarn is given by the following two equations, respectively:

$$
\begin{gathered}
T_{5}=2 \cos \theta T_{1} e^{\mu \sum_{i=1}^{n} \emptyset_{i}}, \\
N_{5}=\frac{T_{5}}{\mu}\left(e^{\mu \varphi_{i}}-1\right),
\end{gathered}
$$




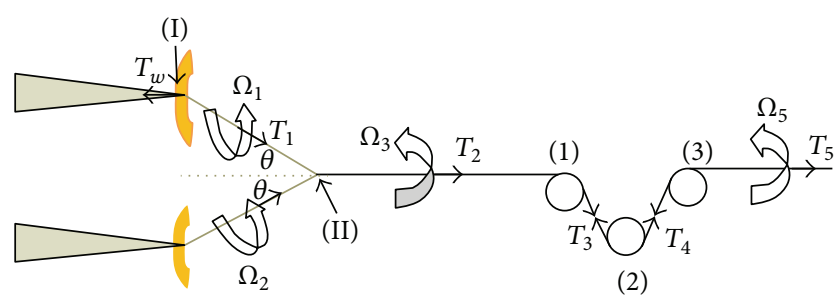

FIGURE 4: Schematic of heating and tension spinning system (HTS) with incorporated tension rods showing related process parameters: $T_{w}, T_{1}, T_{5}, \Omega_{1}$, and $\Omega_{5}$ denoted as the web tension (incoming and outgoing after copper hook), final tension (near the CNT yarn winding spool), twisting of pre-formed thread and twisting of final yarn respectively.

where $\theta$ is the angle between partially twisted thread and the initially formed yarn at the convergent point (near II). Lastly, Figure 4 illustrates various stages in yarn formation leading to the final tension. Alignment and slip occur at the copper hooks, where tension is first applied to the thread. This causes increased CNT interaction in the bundles and yarn. Higher alignment of the CNTs and bundles results in more compact yarn. Therefore, the net effect of the multistage system is to increase the mechanical and electrical properties of the CNT yarn. However, we have observed after a certain maximum tension, further increase in this parameter leads to decreased yarn strength.

3.2. Twisting and Heating Effect on Yarn Formation. For short bundles of CNT drawn from the forest, in the absence of twisting action applied to the yarn, the strength of yarn is limited because of absence of significant transverse forces to bind the CNT assembly together [5]. In order to achieve higher mechanical and electrical properties, the CNTs and bundles need to have maximum contact with one another. This makes twisting a very important part in spinning CNT yarns. At low twist, there is considerable cohesion between the CNTs that can provide reasonable strength to the web. As twisting increases, cohesion also increases, and at a very high level of twisting, brittle behavior is observed [19]. The variation in diameter of CNT yarn is shown in Figures 5(a) to 5(d) at different stages (I to IV) of the HTS process. The SEM images indicate that the diameter of the yarn suddenly decreased as it passed though stage I to stage II (from $14.2 \mu \mathrm{m}$ to $12.1 \mu \mathrm{m}$ ) and then sate II to sate III ( $12.1 \mu \mathrm{m}$ to $10.9 \mu \mathrm{m})$ and remained relatively unchanged afterwards. It was observed from the SEM images (Figures 5(a) to 5(c)) that the twisting angle of the fibers gradually increased with the yarn passing through the stages. We have observed when both heating and tension are applied the diameters of the CNT yarn decreased by about $23 \%$ from 14 to $10.3 \mu \mathrm{m}$ (see Figures 5(a) and 5(d)). SEM images demonstrated that the HTS technique improved the uniformity along the CNT yarn (Figure 5(d)). This technique provides two benefits. First, it increases the alignment of the carbon nanotubes in the thread and also the interbundle lateral cohesion. Second, it reduces the intertube distance and increases the van der Waals force interaction between all the CNTs. Figure 5(e) illustrated the schematic of different positions of heating and tension spinning (HTS) stages are illustrated in Figure 5(f). Raman spectroscopy performed on both CNT yarn formed from treatment 1 and treatment 4 (see Figure 5(f)) do not show important differences that can be readily related to morphological differences in the CNTs for different treatments.

Figure 6(a) shows the resistivity (left $y$-axis) and strength (right $y$-axis) as a function of the tested four different methods of spinning. Substantial improvements in both the electrical and mechanical properties of the threads occurred when applying these methods. Method 4 was carried out using an HTS system temperature of about $225^{\circ} \mathrm{C}$. Figure 6(b) shows that the strength and electrical conductivity of CNT yarn improve $61 \%$ and $71 \%$, respectively, when employing Method 4 as opposed to the Method 1. Method 1 is state of art of spinning yarn which is practiced by most of the research groups [8] and is used in this study as a baseline for comparison. Spinning under tension brings the CNTs in closer contact to each other and therefore enhances the van der Waal interactions. The later causes increased friction between the CNTs, which improves the load transfer between the tubes.

The experimental results showed that treatments $2-4$ brought improvement in the mechanical and electrical properties of the CNT yarns relative to treatment 1 . The highest percentage improvement compared to the baseline treatment 1 was observed for treatments 3 and 4 . This effect is obviously due to applying tension during spinning. However we also noticed that there is a considerable improvement of CNT yarn in treatment 4 compared to treatment 3 . Figures 5 (c) and 5(d) display SEM images of CNT yarns formed from treatment 3 and Method 4. Treatment 4 utilized three tension rods preheated up to $225^{\circ} \mathrm{C}$. It shows that the diameter of the CNT yarn formed from treatment 4 decreases from 10.9 to $10.2 \mu \mathrm{m}$. We assume that the applied simultaneous heating and tension during spinning improve the thread structure by stretching the CNT bundles and enabling more effective thread compactions.

Another interpretation of the results is based on the hypothesis of collapsing the CNTs under tension, which may be enhanced by the applied heating. It was observed by Motta et al. that double walled tubes in yarn corresponding to diameters larger than $\sim 5 \mathrm{~nm}$ collapse into structures with dogbone cross-sections thus forming stacks of parallel graphene layers [7]. The applied tension and radial force change the microscopic structure of the CNTs which tend to polygonize from circular to hexagonal shape.

In the experiments, the mechanical and electrical properties of the CNT yarns substantially improved. This could be due to two reasons. The first reason is the applied heat may have caused evaporation of the absorbed water molecules within the CNT bundles thus increasing the friction and decreasing the air gaps between them. The second is associated with the possible CNT collapse due to the radial pressure incurred from the HTS action. In this scenario stacks of flattened nanotubes can share maximum contact area which will cause increased yarn cohesion. The nanotubes used in this study are double walled with diameters in the range of 8 to $12 \mathrm{~nm}$ which qualifies them for collapsing according to 


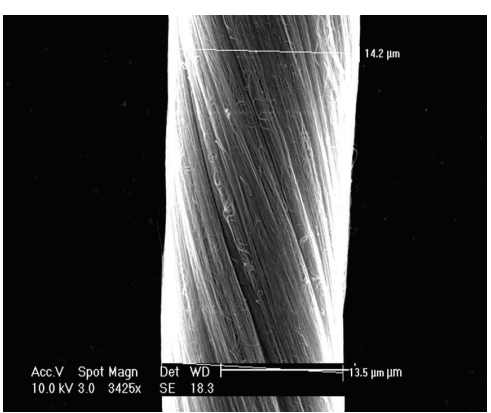

(a)

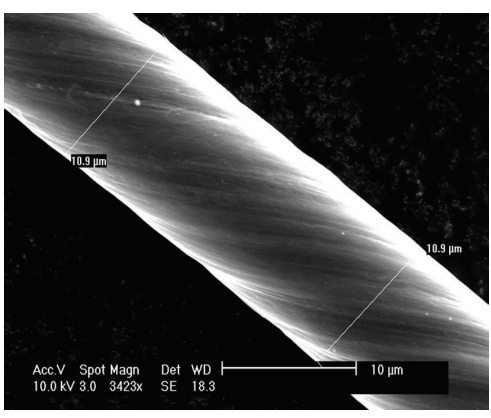

(c)

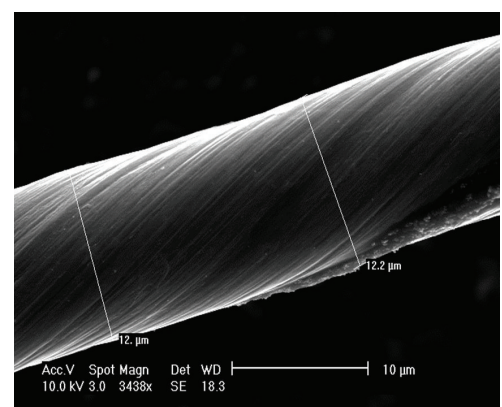

(b)

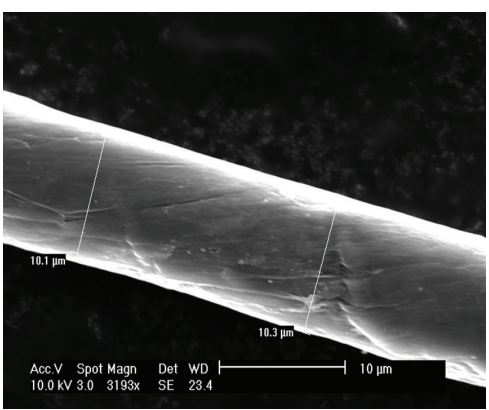

(d)

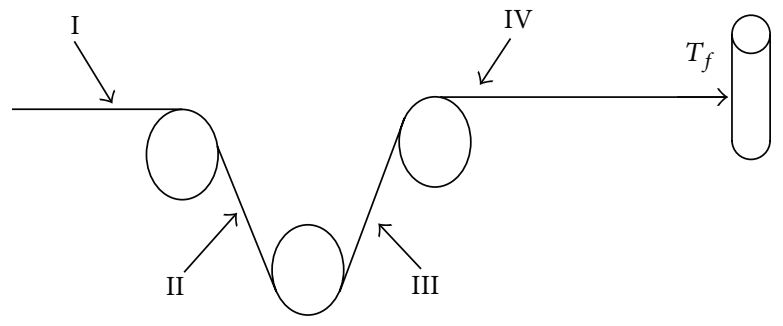

(e)

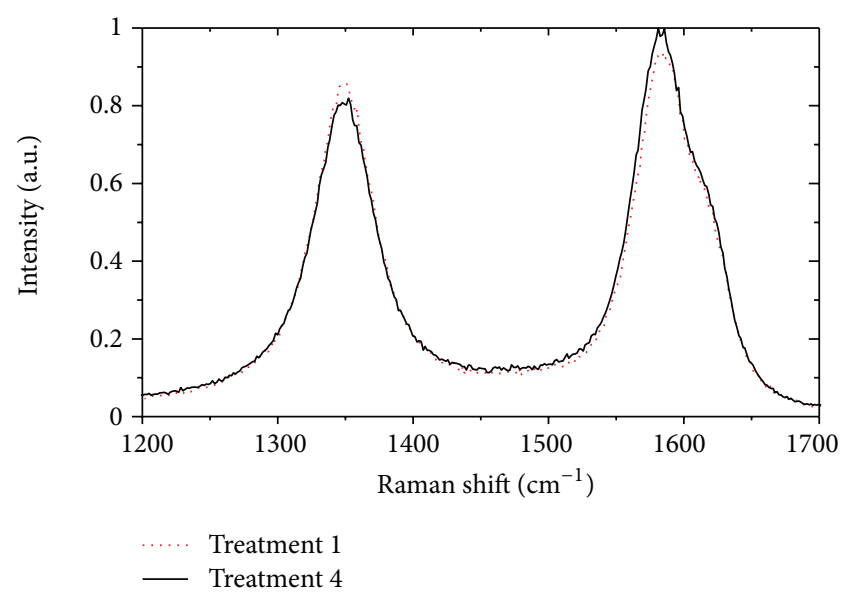

(f)

FIGURE 5: SEM images of CNT yarn at different stages of the spinning process. Stage I (a), Stage II (b), Stage III (c), and Stage IV (d), schematic of the applied heating and tension spinning (HTS) stages (e). I-denotes the position before 1st applied tension; II before 2nd tension; III before 3rd tension; IV before winding on the spool; Raman spectra of CNT yarn formed from treatment 1 and treatment 4(f).

[17]. Thorough High Resolution TEM studies of the CNT yarn may be able to confirm such a phenomenon.

\section{Conclusions}

This work introduced new and improved methods for dry spinning of CNT yarns, which helped to positively affect the yarn structure by stretching the CNT bundles and enabling more effective yarn compaction. The preliminary results for spinning CNT yarn under tension showed an improvement of the fiber mechanical and electrical properties with respect to a standard spinning method used as a baseline. Further enhancement of the CNT yarn quality was observed by introducing a heating and tension spinning (HTS) technique. The tensile strength of the spun yarn when employing HTS improved about $61 \%$ and the electrical conductivity increased by $\sim 71 \%$. Optimization of the new spinning techniques has not been fully achieved and will require further studies to understand the entire temperature and tension effect on the quality of HTS spun yarns. Further optimization of the spinning parameters employing the HTS environment is required, since the results obtained are promising for manufacturing high performance CNT-based yarns. Such experiments are in progress and will be reported in the near future. 


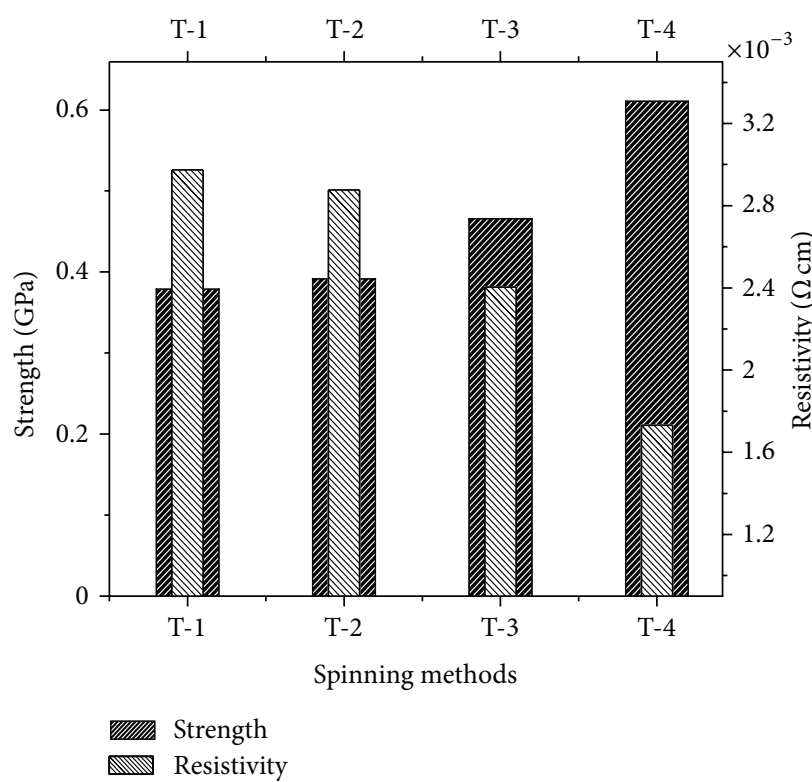

(a)

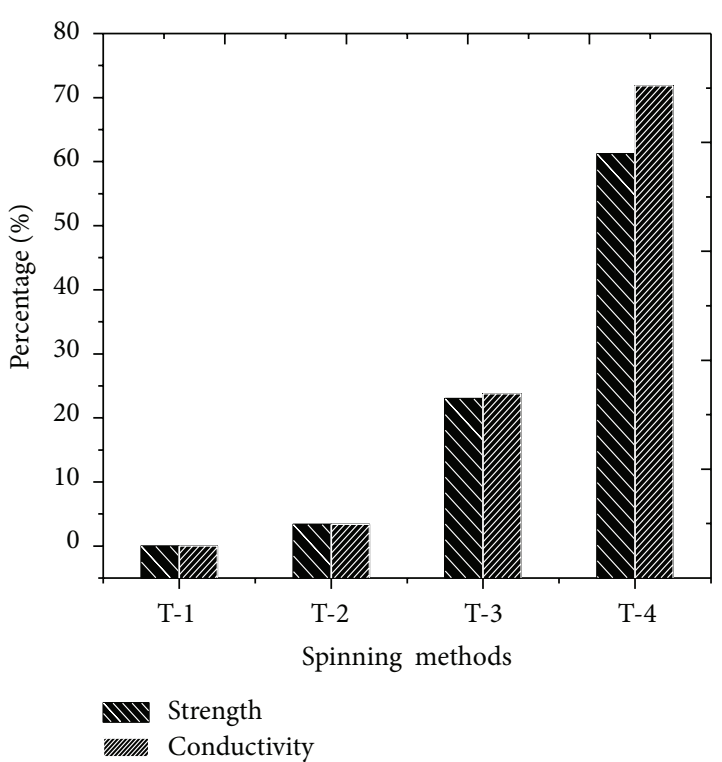

(b)

FIGURE 6: (a) Stress (left $y$-axis) and resistivity (right $y$-axis) versus spinning methods, (b) strength and electrical conductivity improvement in percentage versus spinning methods.

\section{Supporting Data}

See Movie 1 in supplementry material available online at http://dx.doi.org/10.1155/2013/309617.

\section{Acknowledgments}

The authors would like to acknowledge Dr. Sergey Yarmolenko and Svetlana Fialkova from North Carolina A\&T State University for providing instrumentation to test the mechanical properties of the CNT threads and Mr. Doug Hurd for helping to design and manufacture the spinning machine. The financial support from NSF through Grant CMMI07272500 program officer Dr. Haris Doumanidis and from NCA\&T through the ONR is highly appreciated.

\section{References}

[1] L. M. Ericson, H. Fan, H. Peng et al., "Macroscopic, neat, singlewalled carbon nanotube fibers," Science, vol. 305, no. 5689, pp. 1447-1450, 2004

[2] M. E. Kozlov, R. C. Capps, W. M. Sampson, H. Von Ebron, J. P. Ferraris, and R. H. Baughman, "Spinning solid and hollow polymer-free carbon nanotube fibers," Advanced Materials, vol. 17, no. 5, pp. 614-617, 2005.

[3] B. Vigolo, A. Penicaud, C. Coulon et al., "Macroscopic fibers and ribbons of oriented carbon nanotubes," Science, vol. 290, no. 5495, pp. 1331-1334, 2000.

[4] X. Zhang, Q. Li, Y. Tu et al., "Strong carbon-nanotube fibers spun from long carbon-nanotube arrays," Small, vol. 3, no. 2, pp. 244-248, 2007.

[5] H. W. Zhu, C. L. Xu, D. H. Wu, B. Q. Wei, R. Vajtai, and P. M. Ajayan, "Direct synthesis of long single-walled carbon nanotube strands," Science, vol. 296, no. 5569, pp. 884-886, 2002.
[6] M. Motta, A. Moisala, I. A. Kinloch, and A. H. Windle, "High performance fibres from "dog bone" carbon nanotubes," $A d$ vanced Materials, vol. 19, no. 21, pp. 3721-3726, 2007.

[7] M. Motta, Y. L. Li, I. Kinloch, and A. Windle, "Mechanical properties of continuously spun fibers of carbon nanotubes," Nano Letters, vol. 5, no. 8, pp. 1529-1533, 2005.

[8] R. H. Baughman, A. A. Zakhidov, and W. A. De Heer, "Carbon nanotubes-the route toward applications," Science, vol. 297, no. 5582, pp. 787-792, 2002.

[9] K. Koziol, J. Vilatela, A. Moisala et al., "High-performance carbon nanotube fiber," Science, vol. 318, no. 5858, pp. 1892-1895, 2007.

[10] M. Zhang, K. R. Atkinson, and R. H. Baughman, "Multifunctional carbon nanotube yarns by downsizing an ancient technology," Science, vol. 306, no. 5700, pp. 1358-1361, 2004.

[11] Q. Li, X. Zhang, R. F. DePaula et al., "Sustained growth of ultralong carbon nanotube arrays for fiber spinning," Advanced Materials, vol. 18, no. 23, pp. 3160-3163, 2006.

[12] X. Zhang, Q. Li, T. G. Holesinger et al., "Ultrastrong, stiff, and lightweight carbon-nanotube fibers," Advanced Materials, vol. 19, no. 23, pp. 4198-4201, 2007.

[13] C. Feng, K. Liu, J. S. Wu et al., "Flexible, stretchable, transparent conducting films made from superaligned carbon nanotubes," Advanced Functional Materials, vol. 20, no. 6, pp. 885-891, 2010.

[14] M. Naraghi, T. Filleter, A. Moravsky, M. Locascio, R. O. Loutfy, and H. D. Espinosa, "A multiscale study of high performance double-walled nanotube-polymer fibers," ACS Nano, vol. 4, no. 11, pp. 6463-6476, 2010.

[15] S. Fang, M. Zhang, A. A. Zakhidov, and R. H. Baughman, "Structure and process-dependent properties of solid-state spun carbon nanotube yarns," Journal of Physics Condensed Matter, vol. 22, no. 33, Article ID 334221, 2010.

[16] C. Jayasinghe, S. Chakrabarti, M. J. Schulz, and V. Shanov, "Spinning yarn from long carbon nanotube arrays," Journal of Materials Research, vol. 26, pp. 1-7, 2011. 
[17] L. Hu, D. S. Hecht, and G. Grüner, "Infrared transparent carbon nanotube thin films," Applied Physics Letters, vol. 94, no. 8, Article ID 081103, 2009.

[18] J. H. Jung, T. J. Kang, and J. R. Youn, "Effect of bending rigidity on the capstan equation," Textile Research Journal, vol. 74, no. 12, pp. 1085-1096, 2004.

[19] S. Hutton, C. Skourtis, and K. Atkinson, "Tensile and electrical properties of carbon nanotube yarns and knitted tubes in pure or composite form," International Journal of Technology Transfer and Commercialisation, vol. 7, no. 2-3, pp. 258-264, 2008. 

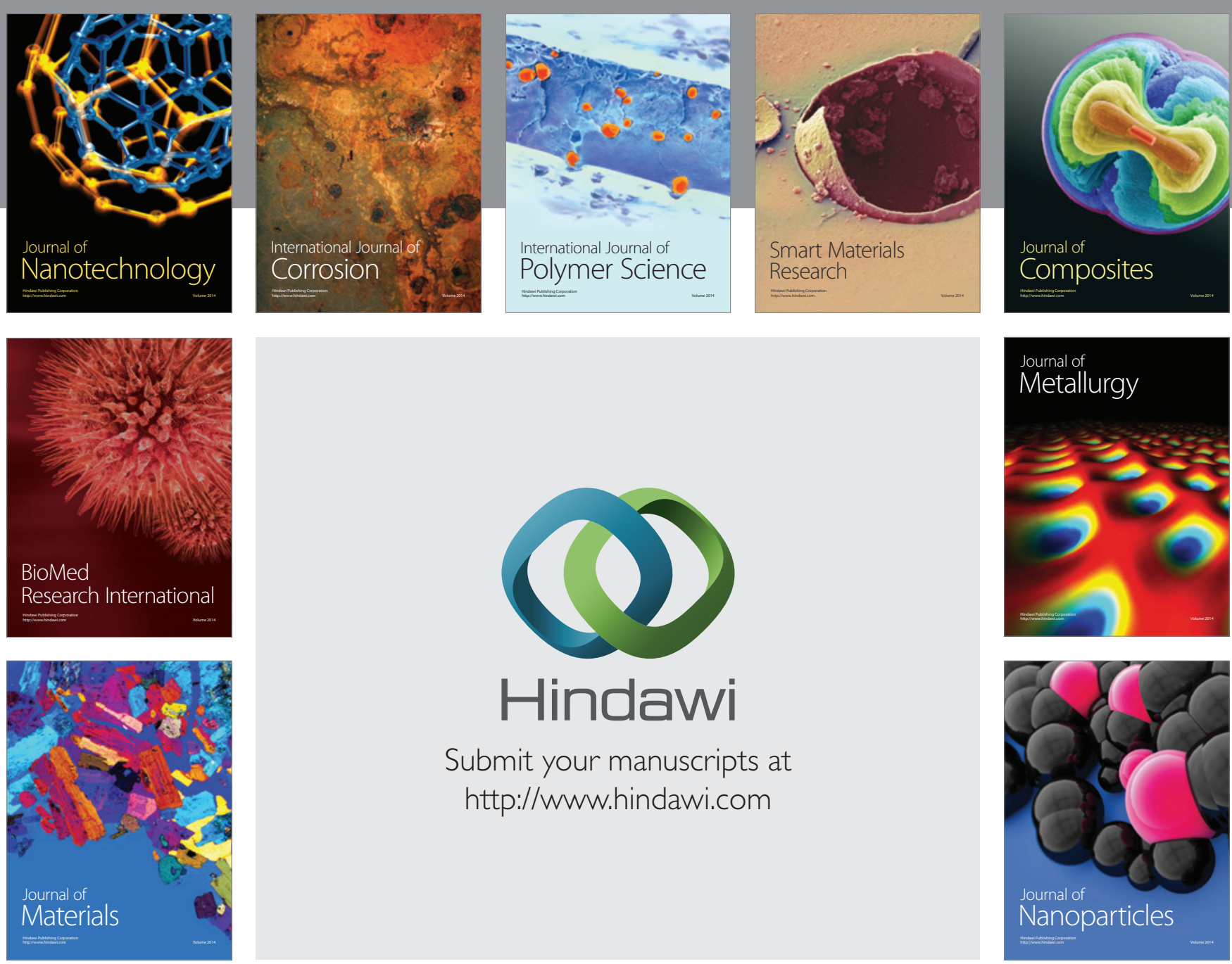

Submit your manuscripts at http://www.hindawi.com
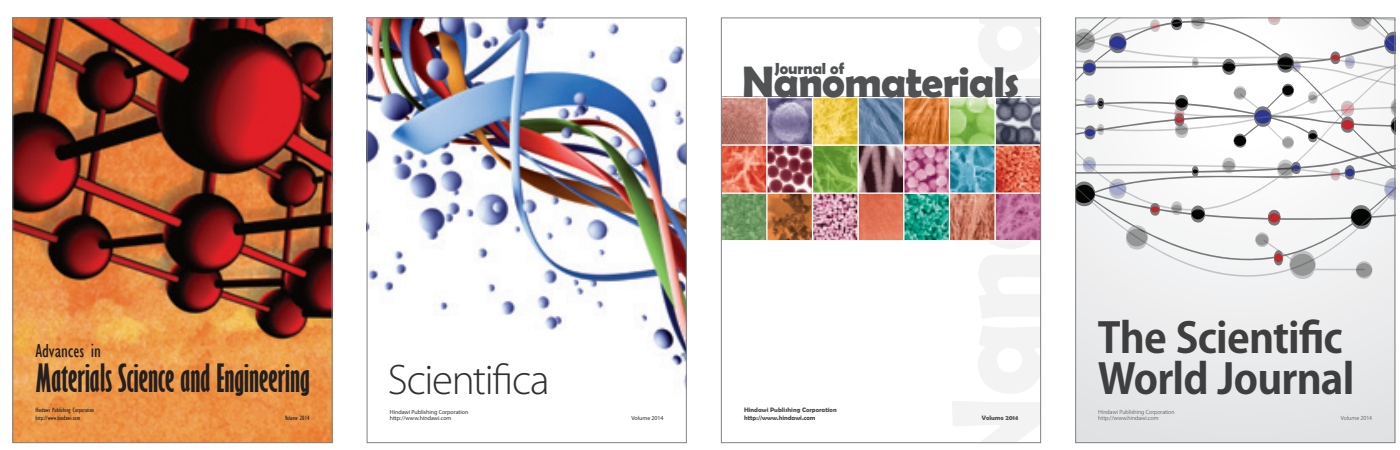

\section{The Scientific World Journal}
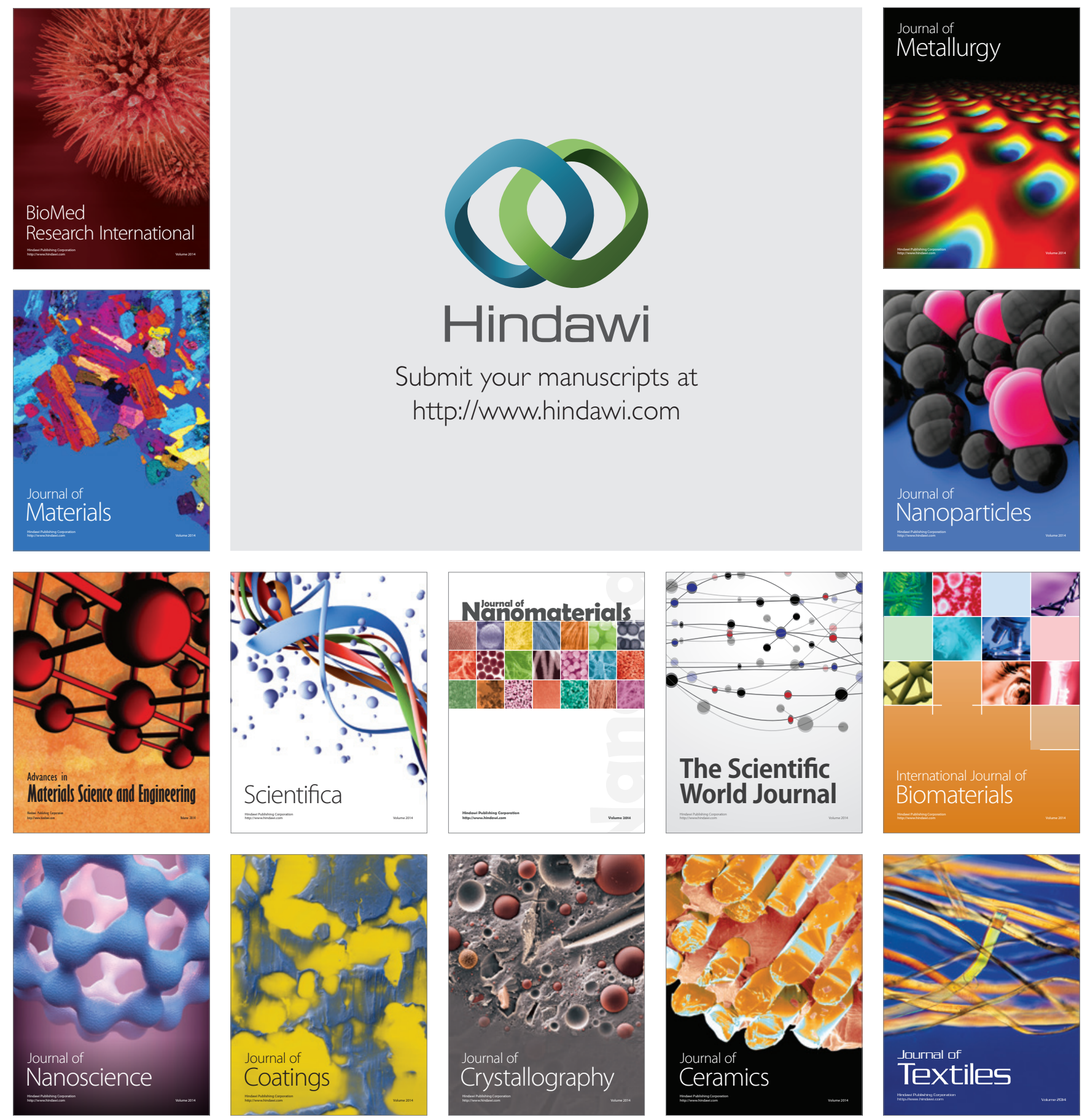